\title{
Modelling of flow behaviour and dynamic recrystallization during hot deformation of MS-W 1200 using the phase field framework
}

\author{
Jan Hiebeler ${ }^{1, a}$, Kirill Khlopkov ${ }^{1}$, Oleg Shchyglo², Thomas Pretorius ${ }^{1}$, Ingo Steinbach ${ }^{2}$ \\ ${ }^{1}$ ThyssenKrupp Steel Europe, Department of modelling and simulation, 47166 Duisburg, Germany \\ ${ }^{2}$ Interdisciplinary Centre for Advanced Materials Simulation (ICAMS), Ruhr-Universität Bochum, Universitätsstr. 150, Germany
}

\begin{abstract}
A new simulation environment is developed to simulate the evolution of microstructure and the corresponding flow stress during rolling. An orientation dependent crystal plasticity hardening model is coupled to grain evolution-, recovery- and recrystallization kinetics within a phase field framework. Hardening and softening kinetics are treated consecutive to differentiate between individual effects. Simulation results are compared to hot compression tests at $1373 \mathrm{~K}$ with a strain rate of $1 \mathrm{~s}^{-1}$.
\end{abstract}

\section{Introduction}

In order to predict material properties after hot forming of steel, it is necessary to understand the microstructural processes during forming. The demand for microstructure simulations is driven by ecological and economic aspects. The first existing simulations were focused on empirically predicting experimental measurable parameters which characterize the material [1-3]. Following the transition towards more physical based approaches leads to an understanding of the importance of a defined microstructure. With the ability to model complex structures today the phase field method is a well-established way to approach the field of thermomechanical microstructure evolution [4-9]. However to successfully setup a simulation environment it is necessary to differentiate between single effects to weigh the influence.

In this work four major modules were coupled to model the hot forming process: I) Crystal plasticity, II) Recovery, III) Recrystallization, IV) Grain evolution.

\section{Experimental section}

In order to determine crystal plasticity input parameters hot compression test of MS-W 1200 steel at T $=1373 \mathrm{~K}$ and deformation rate $\dot{\varphi}=1 \mathrm{~s}^{-1}$ were performed on a deformation dilatometer Bähr DIL 805 D. The decrease of the flow stress shown in Fig. 1 after the true strain reaches approx. 0.4, indicate the presence of dynamic recrystallization. Initial austenite grain size was measured by microscopic analysis after quenching and an annealing treatment at $\mathrm{T}_{\text {anneal }}=823 \mathrm{~K}$ for $900 \mathrm{~s}$. Resulting microstructure analysis shown in Fig. 2 lead to an average grain diameter of $\bar{d}=35 \mu \mathrm{m}$ [10].

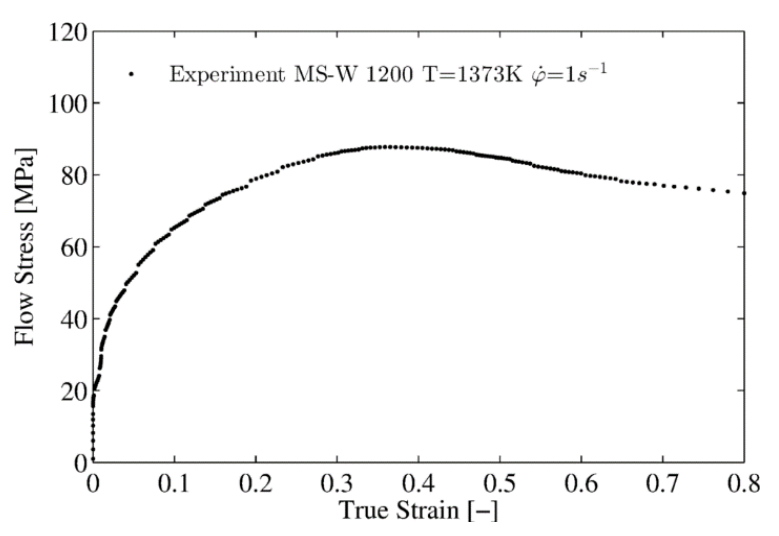

Figure 1. Results of hot compression experiment at $\mathrm{T}=1373 \mathrm{~K}$ and deformation rate $\dot{\varphi}=1 \mathrm{~s}^{-1}$.

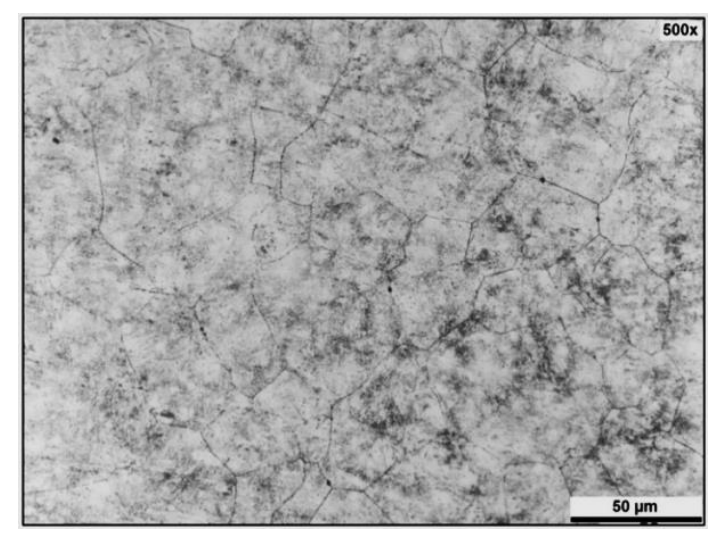

Figure 2. Initial grain structure with average grain size $\bar{d}=35$ $\mu \mathrm{m}[10]$.

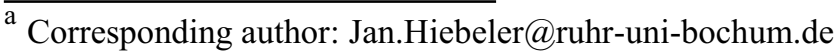




\section{Modelling section}

\subsection{Crystal plasticity}

The crystal plasticity kinetics is based on a model from Hutchinson [11-13] and were incorporated into the phase field framework by Borukhovich and Engels [14]. Starting with the approach by Borukhovich and Engels in this study we implemented the physically based recovery model by Verdier et al. [15] in order to differentiate between single effects. The different phenomena in question are illustrated in Fig. 3.

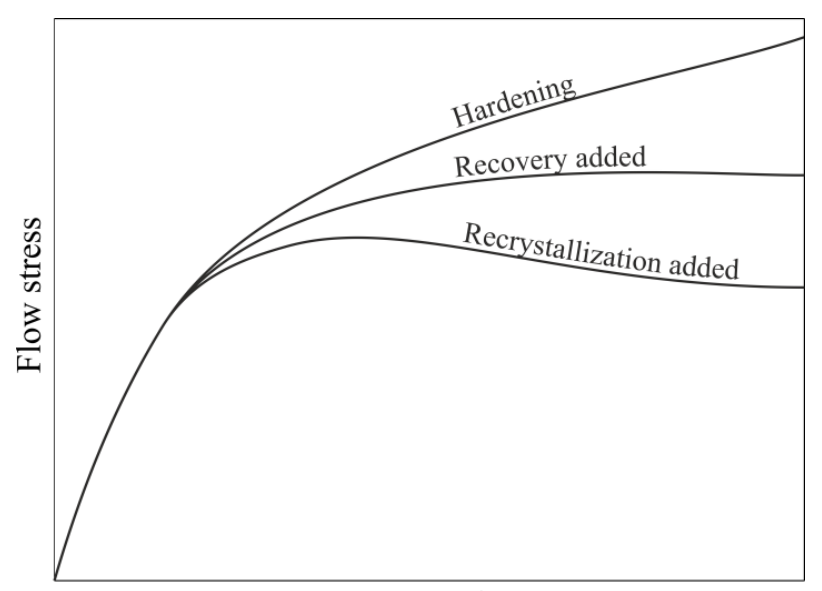

True strain

Figure 3. Differentiation of microstructure evolution effects.

In this study we use the stress dependent shear rate which is given by:

$\dot{\gamma}=\dot{\gamma}_{0} \cdot\left(\frac{\tau}{\tau_{c}}\right)^{\text {Fpow }}$,

with the initial glide velocity $\dot{\gamma}_{0}$, shear stress $\tau$, critical shear stress $\tau_{c}$ and hardening exponent Fpow. The corresponding hardening rate is given by:

$\dot{\tau}_{c}=H_{0} \cdot|\dot{\gamma}|$,

with initial hardening parameter $H_{0}$ and shear rate $|\dot{\gamma}|$. The corresponding dislocation density is given by the Taylor relation [16]:

$\rho=\left(\frac{\tau}{\alpha \cdot \mu \cdot b}\right)^{2}$,

where $\alpha$ is the Taylor factor, $\mu$ is the shear modulus and $\boldsymbol{b}$ is the Burgers vector.

\subsection{Dynamic recovery}

The dynamic recovery model is based on the idea of treating the stress relaxation as an effect of thermally activated annihilation and reorganization of dislocations. The model is derived by expressing the strain rate due to the Orowan relation together with the temperature driven activation criterion for dislocation movement [15]:

$\dot{\tau}=\frac{-E \rho b^{2} v_{D}}{M} \exp \left(\frac{U_{0}}{k_{B} T}\right) \sinh \left(\frac{V_{a c t}}{k_{B} T}\right)$,

with the bulk modulus $E$, Debye frequency $v_{D}$, Taylor factor $M$, activation energy $U_{0}$, activation volume $V_{a c t}$, Boltzmann constant $k_{B}$ and temperature $T$.

\subsection{Dynamic recrystallization}

The dynamic recrystallization model is divided into two parts: I) nucleation and II) growth.

While the classical nucleation theories consider energy as key factor for the onset of dynamic recrystallization [1720] a different approach from [21] introduces a minimum amount of work necessary. By identifying the stored deformation energy as work the authors of [22-24] also incorporate the idea of a relaxation as dissipation part and relate nucleation to the rate of hardening. When an external applied work $\sigma_{c} \dot{\varepsilon}$ exceeds the internal entropy production, $P_{d}^{c}$, another process within the microstructure has to take place which supposed to be the nucleation of new grains with the condition:

$\sigma_{c} \dot{\varepsilon}>P_{d}^{c}$

To catch the exact moment of nucleation one has to analyze the hardening rate over the flow stress where the inflection point indicates the nucleation moment as shown in Fig 4. The advantage lies in the connection to the crystal plasticity kinetic which leads to an implicit orientation dependent recrystallization criterion that is not based on a fixed value but on the individual hardening of each grain within the microstructure.

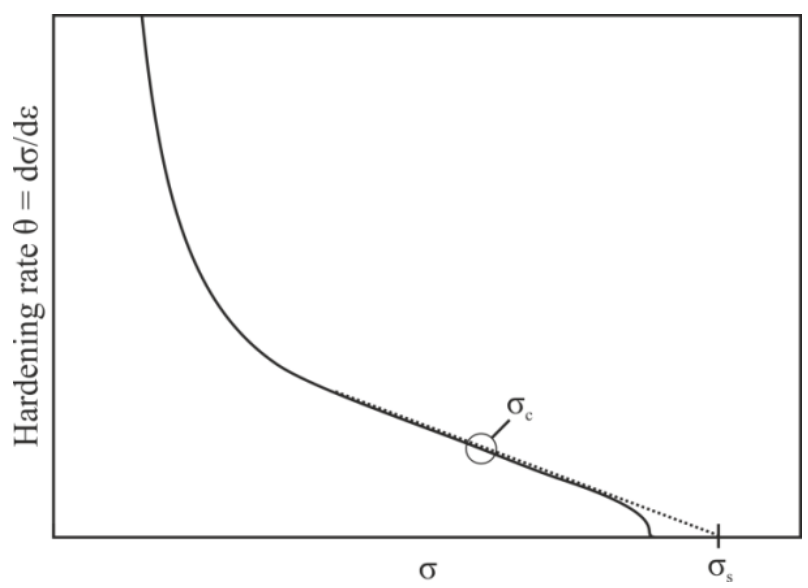

Figure 4. Schematic illustration of the nucleation criterion for dynamic recrystallization from Poliak [22]. 
While a new nucleus is created, it exhibits a crystal orientation. In order to take the line of least resistance the most energetically favoured orientation, related to the local stress field and elastic anisotropy, is chosen. The energy distribution for a uniaxial local stress field compared to the crystal orientation is illustrated in Fig. 5. In our test case the minimal elastic energy is established for an alignment of the crystal orientation towards $\left[\begin{array}{lll}1 & 0 & 0\end{array}\right]$ direction.

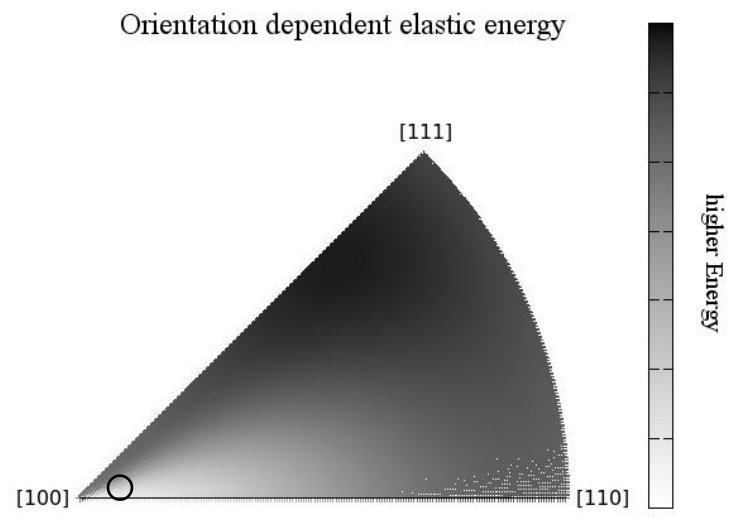

Figure 5. Different crystal orientations and the relating local energy for a uniaxial stress fields. Favoured orientation is indicated by the encircled area.

Subsequently to model grain structure evolution an approach from Beck was implemented which states that grains grow faster towards higher deformation areas [25]. This effect is schematically displayed in the following Figure.

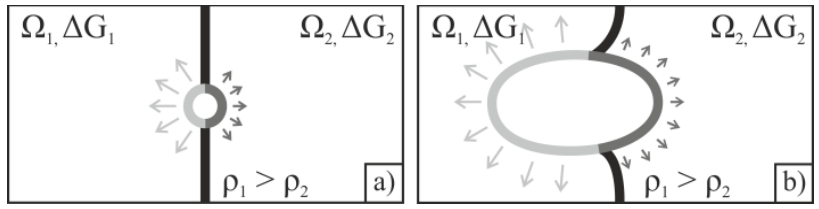

Figure 6. Schematic illustration of the stored energy based boundary migration by [25].

The grain growth velocity is determined by driving the force $\Delta G$ and mobility $m$ :

$v_{g b}=\Delta G \cdot m$

while the driving force results from the difference of stored energies at the grain boundary:

$\Delta G=\gamma_{\alpha}-\gamma_{\beta}$,

with the grain energies $\gamma_{\alpha}$ and $\gamma_{\beta}$ being calculated from stored deformation energy $\gamma_{d}$, shear modulus $\mu$, burgers vector $\boldsymbol{b}$, local volume element $V$ and dislocation density $\rho$ :

$\gamma_{d}=\frac{1}{2} \mu b^{2} \frac{1}{V} \int_{V} \Delta \rho d x$

\section{Simulation and Results}

The simulation was optimized for reasonable simulation time and resolution. The initial grain structure is generated by the OpenPhase code with normal grain size distribution and consists of fourteen grains with different orientations and average grain diameter of $\bar{d}=35 \mu \mathrm{m}[26,27]$. The simulation geometry input parameters are given in Tab. 1. The input parameters for the mechanical solver are given in Tab. 2. These parameters cover elasticity and crystal plasticity at the experimental temperature $\mathrm{T}=1373 \mathrm{~K}$ and strain rate $\dot{\varphi}=$ $1 \mathrm{~s}^{-1}$. Elastic constants were taken from a reference data collection of Ledbetter and Reed [28]. Plasticity related input was derived from hot compression experiments and the dislocation glide velocity was estimated by a work of Güvenc [9]. Recovery kinetics is characterized by the input parameters shown in Tab. 3. Microstructure evolution and recrystallization kinetics input is given in Tab. 4. The simulation procedure is illustrated in the flowchart in the appendix B. Within each time increment of the simulation the four key modules: I) crystal plasticity, II) recovery, III) recrystallization and IV) grain evolution are called. It is important to note that in our study there are no distinct generations of new grains but rather a continuous nucleation and growth of new grains as long as the nucleation and growth conditions are met. Whether a grain grows or vanishes, forms a specific shape or size is not predetermined, but a result of the systems energy minimization.

\subsection{Microstructure evolution}

In Fig. 8 the microstructure evolution during the deformation simulation is shown. The color scale indicates the hardening and the shape of the grain boundaries is illustrated by the gray lines. At first for a deformation of $\varphi=0.12$ the initial grain structure can clearly be identified. Small inhomogeneity of hardening between different grains is observed with an increased hardening at the grain boundary areas. The deformation process continues for $\varphi=0.25$ with increasing hardening inhomogeneity. Overall hardening is the dominant factor in the earlier state of deformation while grains get compressed in loading direction and stretched in orthogonal directions which can be clearly seen by comparing the simulation results for $\varphi=0.12$ and $\varphi=$ 0.40 . For the latter it is notable that although no recrystallization can be observed, there are already several nuclei present in the microstructure, which do influence the evolution, but are not yet resolved in the figure. With further deformation and growth of the nuclei, the recrystallization and corresponding softening process can be seen at $\varphi=0.52$. Several grains have 
evolved from the substructure and contribute to the grain growth process. The softening effect starts to level out during ongoing deformation due to the dynamic recrystallization and growth of already recrystallized but further deformed grains at $\varphi=0.65$ and reaches its plateau at $\varphi=0.80$.

In order to compare the simulation and experimental results the evolution of the flow stress is derived by a homogenization of the critical resolved shear stress over the simulation volume to model the global evolution. The comparison of flow stress evolution between the hot compression experiment and the simulation of MS-W 1200 steel at $\mathrm{T}=1373 \mathrm{~K}$ and strain rate $\dot{\varphi}=1 \mathrm{~s}-1$ is shown in Fig. 7. It is important to note that the obtained flow stress evolution is the result of complex interaction between microstructure evolution and elasto-plastic deformation.

\section{Conclusions}

A consistent full-field model for hot deformation of the steel MS-W 1200 was developed to capture the effects of crystal plasticity, dynamic recovery, dynamic recrystallization and grain evolution. Incorporated into the phase field framework it is possible to keep track of the microstructure evolution during forming. Hot compression experiments were used to identify initial crystal plasticity parameters. Microscopic analysis was used to parametrize the initial grain structure. The simulated flow stress evolution results show good agreement with the experiment.

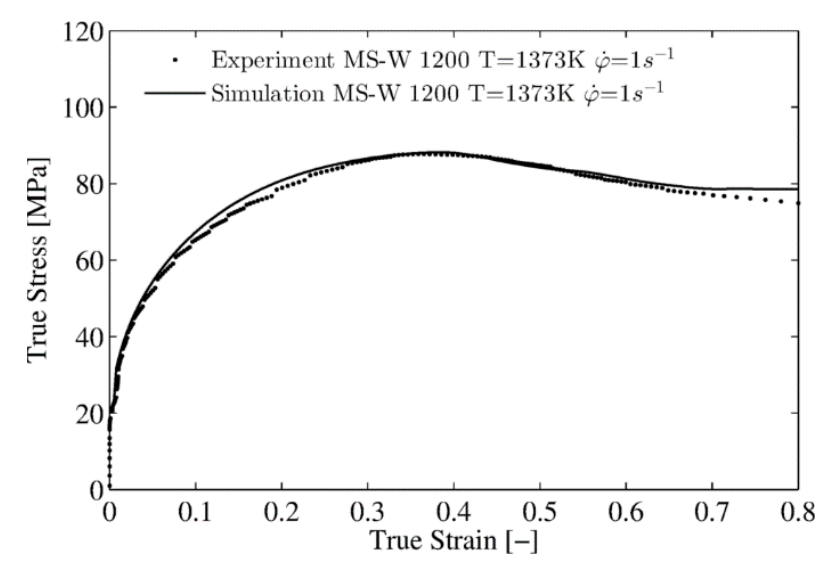

Figure 7. Comparison of flow stress evolution between experiment and simulation of MS-W 1200 steel at $\mathrm{T}=1373 \mathrm{~K}$ and strain rate $\dot{\varphi}=1 \mathrm{~s}^{-1}$.

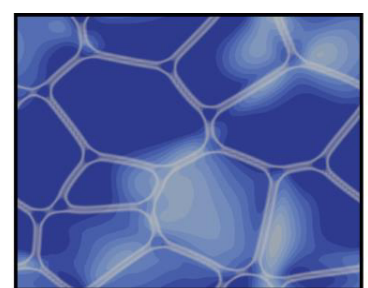

$\varphi=0.12$
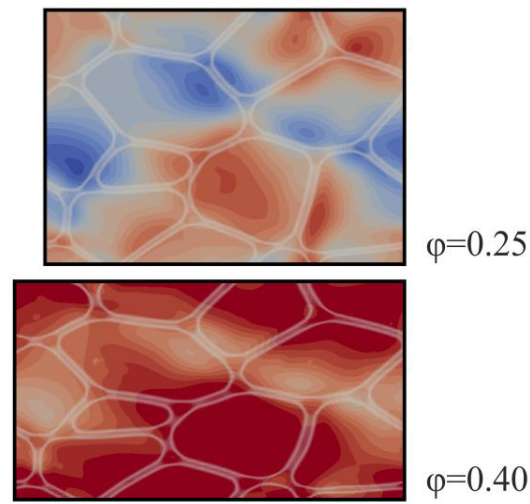

$\varphi=0.25$

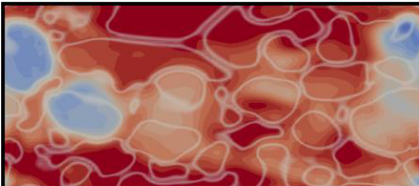

$\varphi=0.40$

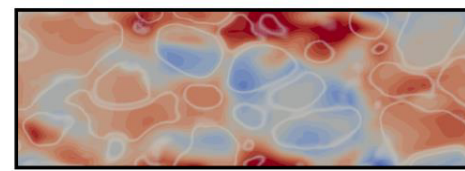

$\varphi=0.52$

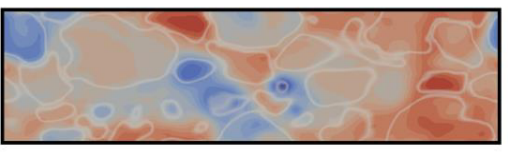

$\varphi=0.65$

$\varphi=0.80$

Critical resolved shear stress $6.3 \mathrm{e} 7$

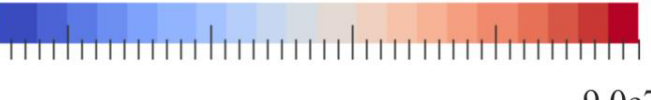

$9.0 \mathrm{e} 7$

Figure 8. Simulation results of microstructure evolution of MS$\mathrm{W} 1200$ steel at $\mathrm{T}=1373 \mathrm{~K}$ and strain rate $\dot{\varphi}=1 \mathrm{~s}^{-1}$. 


\section{References}

[1] M. Avrami, "Kinetics of phase change. III: Granulation, Phase Change and Microstructure," Journal of Chemical Physics, pp. 177-184, 1941.

[2] A. N. Kolmogorov, "Statistical theory of crystallization of metals," Izvestia Akademia Nauk SSSR Ser. Mathematica, pp. 355-359, 1937.

[3] W. A. Mehl and R. F. Johnson, "Reaction Kinetics in processes of nucleation and growth," Transactions of American Institute of Minin and Metallurgical Engineers, pp. 416-458, 1939.

[4] I. Steinbach and O. Shchyglo, "Phase-field modelling of microstructure evolution in solids: Perspectives and challenges," Current Opinion in Solid State and Materials Science, pp. 87-92, 2011.

[5] I. Steinbach, "Phase-Field Model for Microstructure Evolution at the Mesoscopic Scale," 01 Jan, pp. 89-107, 2013.

[6] L. Q. Chen, "Phase-field models for microstructure evolution," Annu. Rev. Mater. Res, 01 Jan, 2002.

[7] M. Muramatsu, Y. Aoyagi, Y. Tadano, and K. Shizawa, "Phase-field simulation of static recrystallization considering nucleation from subgrains and nucleus growth with incubation period," Computational Materials Science, 01 Jan, pp. 112-122, 2014.

[8] N. Moelans, B. Blanpain, and P. Wollants, “An introduction to phase-field modeling of microstructure evolution Comput. Coupling Phase Diagr," Thermochem, 01 Jan, 2008.

[9] O. Güvenc, M. Bambach, and G. Hirt, "Coupling of Crystal Plasticity Finite Element and Phase Field Methods for the Prediction of SRX Kinetics after Hot Working," steel research international, 01 Jan, pp. 999-1009, 2014.

[10] T. J. Baron, K. Khlopkov, T. Pretorius, D. Balzani, D. Brands, and J. Schröder, "Modeling of Microstructure Evolution with Dynamic Recrystallization in Finite Element Simulations of Martensitic Steel," Steel research, 01 Jan, 2015.

[11] D. Peirce, A. Asaro, and R. J. Needleman, "An analysis of non-uniform and localized deformation in ductile single crystals," Acta Metallurgica, 01 Jan, pp. 1087-1119, 1982.

[12] J. W. Hutchinson, "Elastic-Plastic Behaviour of Polycrystalline Metals and Composites," Proc. R. Soc. Lond. A, 01 Jan, pp. 247-272, 1970.

[13] J. Pan and R. J. R, "Rate Sensitivity of Plastic Flow and Implications for Yield Surface Vertices," International Journal of Solids and Structures, 01 Jan, pp. 973-987, 1983.

[14] E. Borukhovich, P. S. Engels, T. Böhlke, and O. Shchyglo, "Large strain elasto-plasticity for diffuse interface models," Modelling and Simulation in Materials Science and Engineering, 01 Jan, 2014.

[15] M. Verdier, Y. Brechet, and P. Guyot, "Recovery of AlMg Alloys: Flow Stress and Strain-
Hardening Properties," Acta Materialia, 01 Jan, 1999.

[16] G. I. Taylor, "The Mechanism of Plastic Deformation of Crystals. Part I. Theoretical," Proceedings of the Royal Society of London, 01 Jan, pp. 362-387, 1934.

[17] C. Zener and J. H. Hollomon, "Effect of Strain Rate Upon Plastic Flow of Steel," J. Appl. Phys, 01 Jan, 1944.

[18] R. D. Doherty, D. A. Hughes, F. J. Humphreys, J. J. Jonas, J. J. D, M. E. Kassner, W. E. King, T. R. McNelley, H. J. McQueen, and A. D. Rollett, "Current issues in recrystalliszation: a review," Material Science and Engineering, 01 Jan, pp. 219-274, 1997.

[19] F. J. Humphreys and M. Hatherly, Recrystallization and related Annealing Phenomena: Pergamon, 1995.

[20] C. M. Sellars and J. A. Whiteman, "Recrystallization and Grain Growth in Hot Rolling," Metall Science, 01 Jan, pp. 187-194, 1997.

[21] P. J. Wray, "On set of recrystallization during the tensile deformation of austenitic iron at intermediate strain rates," Metall. Trans, 01 Jan, pp. 1197-1203, 1975.

[22] E. I. Poliak and J. J. Jonas, "A one-parameter approach to determining the critical conditions for the initiation of dynamic recrystallization," Acta Materialia, 01 Jan, 1996.

[23] J. Kestin, Article in Patterns, Defects and Microstructures in Non-Equilibrium Systems: Martinus Nijhoff, 1987.

[24] M. G. Beghi, C. E. Bottani, and G. Caglioti, "Thermoelastic-Plastic Transition and Dislocation Dynamics in Metals by Deformation Calorimetry,” NATO ASI Series, pp. 187-201.

[25] P. A. Beck and P. R. Sperry, "Strain induced boundary migration in high purity aluminum," Journal of Applied Physics, 01 Jan, pp. 150-152, 1950.

[26] R. D. Kamachali, Grain boundary motion in polycrystalline materials. Bochum, 2012.

[27] I. Steinbach, "Phase-field models in materials science," Modelling Simul. Mater. Sci. Eng, 01 Jan, pp. 1-31, 2009.

[28] H. M. Ledbetter and R. P. Reed, "Elastic Properties of Metals and Alloys," J. Phys. Chem. Ref. Data, 01 Jan, pp. 531-617, 1973. 


\section{Appendix A}

Table 1. Environmental input parameters for simulation.

\begin{tabular}{|c|c|c|c|}
\hline Simulation edge length & - & 100 & $\mu \mathrm{m}$ \\
\hline Average gran diameter & $\bar{d}$ & 35 & $\mu \mathrm{m}$ \\
\hline Simulation resolution & - & $100^{2}$ & data points \\
\hline Simulation increments & $\mathrm{N}$ & 200 & $\#$ \\
\hline Number of grains & - & 14 & $\#$ \\
\hline Boundary conditions & - & \multicolumn{2}{|c|}{ periodic } \\
\hline
\end{tabular}

Table 2. Input parameters for the mechanical solver for

temperature $\mathrm{T}=1373 \mathrm{~K}$ and strain rate $\dot{\varphi}=1 \mathrm{~s}^{-1}$.

\begin{tabular}{|c|c|c|c|}
\hline \multirow{2}{*}{ Anisotropic elastic constants } & $\mathrm{C} 11$ & $160 \cdot 10^{9}$ & $\mathrm{~Pa}$ \\
\cline { 2 - 4 } & $\mathrm{C} 12$ & $120 \cdot 10^{9}$ & $\mathrm{~Pa}$ \\
\cline { 2 - 4 } & $\mathrm{C} 44$ & $100 \cdot 10^{9}$ & $\mathrm{~Pa}$ \\
\hline $\begin{array}{c}\text { Initial critical resolved shear } \\
\text { stress }\end{array}$ & $\mathrm{T}_{\mathrm{c}, 0}$ & $20 \cdot 10^{6}$ & $\mathrm{~Pa}$ \\
\hline $\begin{array}{c}\text { Strain rate } \\
\text { Exponent for flow evolution }\end{array}$ & $\mathrm{F}_{\mathrm{pow}}$ & 2.02 & - \\
\hline $\begin{array}{c}\text { Hardening parameter } \\
\text { Burgers vector }\end{array}$ & $\mathrm{H}_{0}$ & $1.1 \cdot 10^{13}$ & - \\
\hline $\begin{array}{c}\text { Shear modulus } \\
\text { Initial dislocation glide } \\
\text { velocity }\end{array}$ & $\mathrm{b}$ & $2.56 \cdot 10^{-10}$ & $\mathrm{M}$ \\
\hline $\mathrm{v}_{\mathrm{o}}$ & $10^{-3}$ & $\mathrm{~ms}^{-1}$ \\
\hline
\end{tabular}

Table 3. Input parameters for the recovery kinetics.

\begin{tabular}{|c|c|c|c|}
\hline Debye frequency & $\mathrm{v}_{\mathrm{D}}$ & $5.4 \cdot 10^{12}$ & $\mathrm{~s}^{-1}$ \\
\hline Taylor factor & $\mathrm{a}$ & 0.3 & - \\
\hline Activation energy & $\mathrm{U}_{0}$ & 327300 & $\mathrm{Jmol}^{-1}$ \\
\hline Activation volume & $\mathrm{V}_{\mathrm{act}}$ & $28 \mathrm{~b}^{3}$ & $\mathrm{~m}^{3}$ \\
\hline Temperature & $\mathrm{T}$ & 1373 & $\mathrm{~K}$ \\
\hline
\end{tabular}

Table 4. Input parameters for the recrystallization and microstructure evolution kinetics.

\begin{tabular}{|c|c|c|c|}
\hline $\begin{array}{c}\text { Mobility of high angle } \\
\text { grain boundaries }\end{array}$ & $\mathrm{m}$ & $1.2 \cdot 10^{-11}$ & $\mathrm{~m}^{4} \mathrm{~J}^{-1} \mathrm{~s}^{-1}$ \\
\hline $\begin{array}{c}\text { Energy of high angle } \\
\text { grain boundary }\end{array}$ & $\gamma_{\mathrm{gb}}$ & 0.6 & $\mathrm{Jm}^{-2}$ \\
\hline $\begin{array}{c}\text { Average energy per unit } \\
\text { length of dislocation }\end{array}$ & $\mathrm{U}_{\mathrm{e}}$ & $1.31 \cdot 10^{-9}$ & $\mathrm{Jm}^{-1}$ \\
\hline $\begin{array}{c}\text { Cut-off radius for } \\
\text { nucleation }\end{array}$ & $\mathrm{R}_{\text {cutoff }}$ & 4 & $\mu \mathrm{m}$ \\
\hline $\begin{array}{c}\text { Mobility factor for } \\
\text { nuclei }\end{array}$ & $\mathrm{C}_{\mathrm{m}}$ & 18.6 & - \\
\hline Burgers vector & $\mathrm{b}$ & $2.56 \cdot 10^{-10}$ & $\mathrm{~m}$ \\
\hline
\end{tabular}




\section{Appendix B}

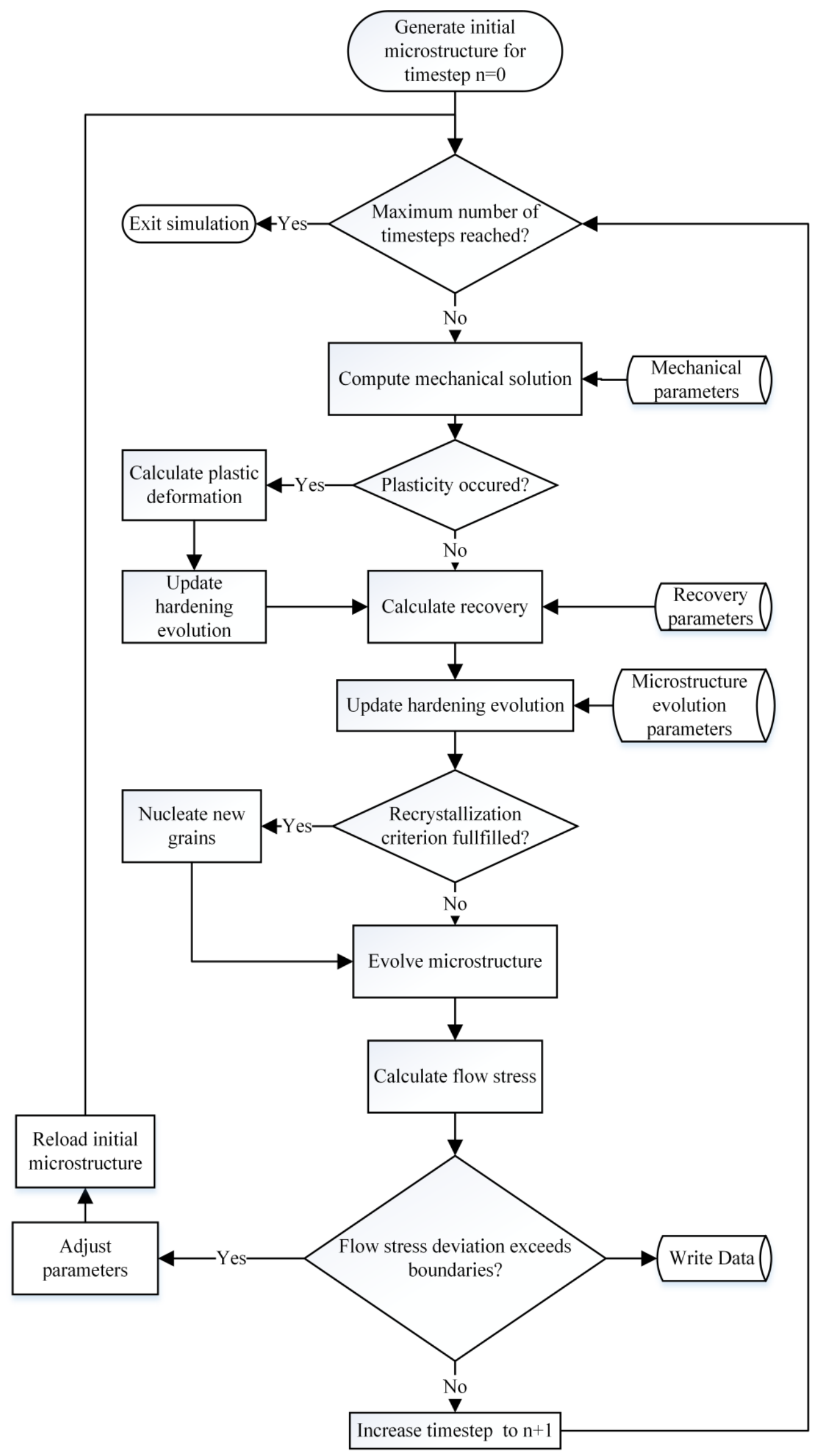

